\title{
Effects of Resource Availability on Consensus Decision Making in Primates
}

\author{
Julian Zappala and Brian Logan \\ School of Computer Science, \\ University of Nottingham, UK \\ $\{j x z$, bsl $\}$ ecs.nott.ac.uk
}

\begin{abstract}
Recently there has been an increasing interest in the group decision making process, and in particular the mechanisms through which a group of individuals can arrive at a consensus decision. In this paper we investigate the effects of resource availability upon consensus decision making in a primate group. We extend an existing agent-based model of primate decision making to include a representation of diminishing foraging returns. Our results indicate that environmental factors, and in particular the rate of energy acquisition from foraging activities, has an impact on successful strategies for consensus decision making in primate groups. The introduction of greater granularity to the environmental model through the inclusion of diminishing returns also results in increased agreement between the model's predictions and data gathered through empirical observation of a naturally occurring primate group.
\end{abstract}

\section{Introduction}

Many non-human animals live in social groups. The advantages of group living, including e.g. decreased risk of predation [1], depend on the group remaining cohesive. However differences in the drives and desires of individuals have the potential to result in group fragmentation, particularly when the group is presented with choice [2]. Yet, groups routinely make decisions regarding which activity to pursue, where to pursue it, the direction of movement and the timing of departure [3] without fragmenting. The mechanisms underlying such consensus decision making are an important but poorly understood aspect of group-level dynamics. Many factors may potentially affect how such decisions are made, including dominance hierarchy and social and familial affiliation [4], the consensus cost (relative to grouping benefits), the physical homogeneity of the group, the size of the group (relative to an optimal size) and the nature of possible activities [5].

Much of the data pertaining to group decision making in animals has been collected through observational field-based research. However, studies of this kind are frequently resource intensive and logistically complex making large scale/long term data collection impractical. It is often infeasible, and generally undesirable, to attempt to conduct behavioural research on animal groups within a laboratory setting; yet without the controls provided by laboratory conditions we lack the consistency and repeatability necessary to draw strong conclusions from experimental findings [6]. Given these difficulties, 
multi-agent simulation offers an attractive alternative to field or laboratory-based studies of group decision making mechanisms. The results of an agent-based model depend on the desires of each individual agent, its current internal state (which may include an internal world model) and the sensory information it receives. This reliance on individual choice makes agent-based modelling especially useful when dealing with animals which live in groups, since it is likely that the optimal strategy for an individual depends on the strategies adopted by others in the group [7]. Agent-based simulation has been successfully applied to the investigation of group decision making in animals across a variety of taxa and scenarios including nest selection in ants [8], shelter selection in cockroaches [9], and action selection in primates [10].

In this paper, we investigate the effect of environmental features, and specifically resource availability on consensus group decision making. We extend an agent-based model of primate group decision making [10] to include a model of diminishing resource returns whereby energetic (food) availability within a region is reduced proportionally to the degree of foraging activity. We study the implications of diminishing returns on the ability of group members to achieve their goals and its implications for group decision making, specifically its impact on the quorum threshold. We show that as the favourability of environmental conditions decreases the value of the quorum threshold becomes more critical to group wellbeing.

The remainder of this paper is organised as follows. In Section 2 we briefly present a theoretical framework for consensus decision making in animal groups. In Section 3 we summarise the agent-based model of consensus decision making in primates developed by Sellers et al. [10] which forms the basis of our work, and highlight some of the limitations of this model. Our model of diminishing resource returns is presented in Section 4 and in Section 5 we summarise the results of a replication and validation study of the Sellers et al. model. In Section 6 we present initial results of experiments with our model of diminishing returns, and compare these with with results from [10] and field observations. In Section 7 we consider the implications of our work in the context of related literature. Finally we consider options for future work and expansion of this study in Section 8 .

\section{Group Decision Making}

For a group of animals to remain cohesive, individuals must synchronise the timing and location of their activities. However, this synchronisation may result in an individual performing a non-optimal activity [2]. Biomechanical and physiological differences between individuals resulting from variations in age, gender, and reproductive status, can lead to divergent abilities and motives, giving rise to conflicting needs amongst group members [2]. In social species the outcome of collective decisions has implications for the fitness of each animal, yet individuals will defer to these decisions even where it may not be in their best interests to do so [5]. Decision making strategies employed by animal groups must maximise the welfare of the group overall without excessively compromising the requirements of each individual [5]. At present, we understand very little about the mechanisms by which such agreement can be reached, in a non-verbal way, and by animals of limited cognitive ability. 
Conradt and Roper [11] define a consensus decision as a choice between two or more mutually exclusive actions with the specific aim of reaching a consensus. All group members must abide by the outcome of this decision, however, it is not required that all group members contribute to the making of the decision. This variation leads to three further sub-classifications; equally shared consensus decision where all members do contribute towards the decision (democratic), partially shared consensus decision where only a proportion of members (e.g., all adult males) are contributors, and unshared consensus decision where the decision is made by a single individual (despotic). The latter form may occur either under consistent leadership where the same individual is always responsible for the decision or variable leadership where different individuals take this responsibility at different times.

For a shared consensus decision to be achieved a quorum, representing a required minimum number of group members, must agree. The quorum may be a sub-majority, a majority, or a super-majority, with thresholds for these being less than fifty percent, at least fifty percent and more than fifty percent of group members respectively. Where the needs of an individual conflict with the outcome of a consensus decision, that individual may bear a consensus cost, for example, to their fitness. Consensus decisions are typically made in respect of synchronised group activities, e.g., timing and direction of travel, and which activities are pursued and where they are pursued [11].

\section{A Model of Consensus Decision Making}

In [10], Sellers et al. present a multi-agent simulation of group decision making in a troop of 50 chacma baboons (Papio hamadryas ursinus), and compare the results of their simulation with data collected over a seven month period of field observation at the De Hoop Nature Reserve in South Africa. In this section, we briefly summarise the Sellers et al. model and key results of their simulation experiments.

The simulation model consists of two components: the environment model and the baboon model. The environment model is based on the $200 \mathrm{~m} \times 200 \mathrm{~m}$ map grid used for field data recording, and consists of 660 cells within an area $5.4 \mathrm{~km}$ by $8.4 \mathrm{~km}$. Each cell contains a mixture of the 6 primary habitat types found at De Hoop (Acacia Woodland, Burnt Acacia Woodland, Climax Fynbos, Burnt Fynbos, Grassland and Vlei) and may also include one or more 'special features': water sources, sleeping sites, and refuges (primarily cliffs). Each habitat type is characterised by a maximum food availability, food intake rate when foraging and travel-foraging, and replenishment rate for each month of the study period.

The baboon model models each baboon as an agent with physical parameters based on well-known baboon physiology. In addition, each agent maintains an individual score for water, energy and social time which function as 'drives' in biasing the agent's choice of preferred activity. At each timestep an agent can perform one of five actions corresponding to the activities recorded for the baboons at De Hoop: drinking, foraging, moving (travel foraging), socialising or resting. Performing an action updates the appropriate scores. For example, foraging updates the agent's energy score with the net energy gain, i.e., the difference between the amount and the type of food consumed (determined by the habitat type(s) of the current cell) and the energy cost of foraging. 
Foraging and travel-foraging also cause the food available in the grid cell containing an agent to be depleted at the appropriate food intake rate for each habitat type occurring in the cell. While food consumed is replaced at the replenishment rate for the current simulation month for each of the habitat type(s) occurring in the grid cell, this is lower than the corresponding food intake rate. Foraging therefore reduces the availability of food at the next timestep (relative to other cells within the agents' perceptual range), making it more likely that an agent will choose to forage in another cell (or perform some other action) at the next timestep.

The model assumes equally shared consensus decision making. Agents, under the constraint that they must not separate, use a democratic voting mechanism to arrive at a consensus decision concerning the direction of group travel. Consensus decisions may result in a consensus cost for some agents, if they are not able to perform their (individually) preferred action as a result of a group decision to move in a particular direction.

Monte Carlo simulation was used to determine how well the model was able to duplicate the observed activity patterns of animals found in the field data, and to estimate the importance of decision parameters on the outcome. (See table 1 for the Monte Carlo parameters.) The quorum threshold $(V)$ was found to be the most important determinant of both the fitness of each agent and the behaviour of the group as a whole. Thresholds of between $50 \%$ and $65 \%$ provided agents with the greatest chances of survival; outside of this narrow band the chances of an agent failing to meet its survival goals, measured in terms of food/water intake and time spent in social engagement, were seen to drastically increase.

However, while the Sellers et al. model successfully meets its requirements in terms of goal achievement in a large proportion of cases, it is less successful in predicting energy budgets and habitat utilisation (see figure 2). The agents spend significantly less time foraging and significantly more time resting than the natural population. A similar pattern is seen in the habitat types used by the agents: the agents use the Vlei habitat significantly more than the natural population, and significantly underutilise the Acacia Woodland habitat. Sellers et al. speculated that the poor fit to the data may be due in part to the agents foraging too easily. Agents uniformly deplete $200 \mathrm{~m} \times 200 \mathrm{~m}$ cells without incurring additional search costs. In reality, local resource depletion occurs on a much finer scale with more rapidly diminishing foraging returns, and this is not captured in the model. In this paper, we extend the Sellers et al. model [10] to include a simple model of the effects of diminishing returns and investigate the implications of diminishing returns for group decision making.

\section{A Model of Diminishing Returns}

We extend the Sellers et al. model by introducing a new parameter, occupancy penalty $(p)$. The occupancy penalty is used to compute the diminished energy resource in a habitat cell as a function of the daily total occupancy time of that cell, according to:

$$
d=\frac{v}{1+(t \times p)}
$$


where $d$ is the (diminished) available energy resource in a habitat cell, $v$ is the available energy resource in the cell in the original Sellers et al. model, and $t$ is the number of time steps that habitat cell has been occupied during the current simulated day. The occupancy penalty is inversely proportional to the half-life of the decay, e.g. an occupancy penalty of 0.1 means that a given constant will decay to half of its value in ten time steps. The effect of the occupancy penalty on a typical habitat cell is illustrated in Figure 1.

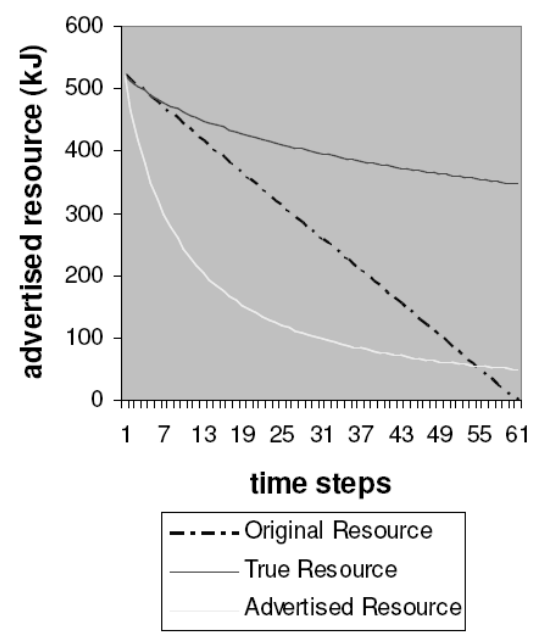

(a) Resource availability

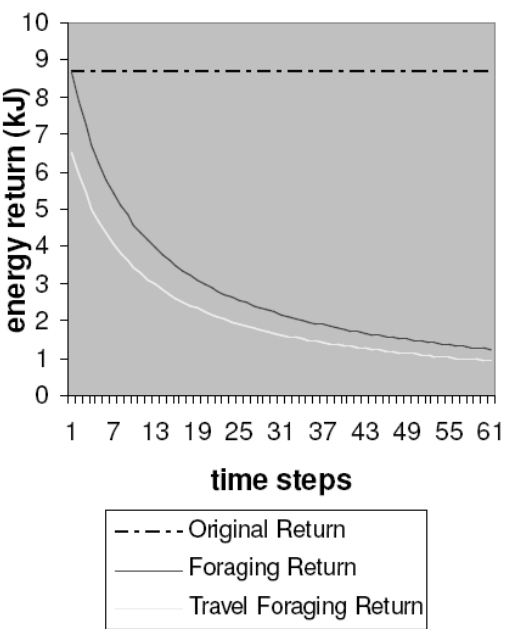

(b) Energy Returns

Fig. 1. Effect of occupancy penalty on resource availability and energy returns. Original Resource and Original Return represent the behaviour of the simulation prior to the introduction of the occupancy penalty. True Resource and Advertised Resource represent the actual resource, and diminished resource of the habitat cell (as perceived by the agent), respectively. Foraging Return and Travel Foraging (foraging whilst on the move) Return represent the net energy returns from these activities after the introduction of the occupancy penalty. $(p=0.1)$

The occupancy penalty serves two purposes: firstly it provides a model of diminishing foraging returns; decreasing the rate at which agents obtain food from a given location as a function of the time spent at that location; secondly, it reduces the availability of food in cells previously occupied during the course of a simulated day.

With the exception of the model of diminishing returns, the revised model is identical in all respects to that of Sellers et al.. 


\section{Simulation Replication \& Validation}

To verify that our revised model was in fact identical to that in [10] we performed a replication and validation study.

Replication is one of the tenets of cumulative science, yet the findings of simulation studies are rarely verified in this manner. However without independent replication, it is possible that the results of simulation experiments are partially or wholly attributable to implementation artefacts or simple programming errors [12]. To validate the results of Sellers et al. the original simulation was re-implemented (without the diminishing returns model) using the MASON simulation toolkit [13]. MASON was chosen for its high performance relative to other Java based toolkits [14]. ${ }^{1}$

A rigorous validation of the replicated simulation was undertaken. To account for stochastic elements within the model an identical stream of random numbers were used, in identical circumstances (isomorphically) [15] whilst executing both original and reimplemented simulations in parallel; this approach permits direct comparison of the complete internal state of both models. The re-implementation was shown to exhibit numerical identity [16] when compared to the original work.

The replication revealed a small number of minor defects in the original implementation relating to choice of direction and group action selection under extremely rare conditions. Deterministic behaviour in the group decision making algorithm could also lead to a bias towards moving in the direction of locations suitable for foraging in cases where a sub majority quorum threshold is applied to the group decision making procedure.

These defects were corrected and the revised and original models were statistically compared using a two-tailed t-test for matched pairs. 1001 sets of parameter values were randomly chosen from the original Monte Carlo parameter ranges used in [10] and the corresponding outputs analysed for each simulation. The models presented distributional equivalence [16] $(n=1001, p<0.05)$ indicating no significant difference in their outputs. We take this as strong indication that these defects do not materially affect the original findings of Sellers et al. The replication also gives us greater confidence that the results presented in [10] were not due to artefacts of the original implementation, and that the results of the diminishing returns model presented below are likewise not due to artefacts in the new implementation.

\section{Results}

The diminishing returns model was then incorporated into the new MASON simulation, and a number experiments performed to determine the effects of diminishing returns relative to the original model (with defects corrected).

Our initial experiments were focussed on calibrating the occupancy penalty. This was achieved through observation of the effects of different values for $p$ on the average times spent in each activity and habitat relative to the original simulation and to the field data. Average times for each activity and habitat were computed for both simulations

\footnotetext{
${ }^{1}$ The source code for the new simulation is available from the authors on request.
} 
for 1001 sets of input parameters randomly sampled from the original Monte Carlo parameters and ranges in [10]. Values used for the Monte Carlo simulations are shown in Table 1, for a complete description of these parameters see [10].

Table 1. Monte Carlo parameters and ranges.

\begin{tabular}{cccc}
\hline Parameter & Meaning & Lower Bound Upper Bound \\
\hline$V$ & Proportion to win vote (quorum threshold) & 0.1 & 0.9 \\
$S$ & Search radius & 200 & 2200 \\
$W_{F}$ & Relative food importance & 1 & 10 \\
$W_{S}$ & Relative socialising importance & 1 & 10 \\
$W_{D}$ & Relative drinking importance & 1 & 10 \\
$T_{F}$ & Foraging move threshold & 4 & 6 \\
$T_{S}$ & Socialising move threshold & 1 & 3 \\
$T_{R}$ & Socialising move threshold & 1 & 3 \\
$T_{K}$ & Resting/socialising risk threshold & 0 & 0.25 \\
\hline
\end{tabular}

We found that even a modest occupancy penalty of $p=0.1$ had a noticeable impact on both the average amount of time the agents spent foraging and moving, increasing the latter well above the observed level. The agents seemingly "preferred" to move more frequently, into cells not previously occupied, than to remain in a habitat cell and forage at the lower rates of return resulting from application of the occupancy penalty. In order to compensate for this effect the range of the Foraging Move Threshold $\left(T_{F}\right)$ parameter was adjusted from between 1 and 3 (as used in [10])to between 4 and 6 (as shown in Table 1). The Foraging Move Threshold determines the degree to which an alternative cell must be better than the current cell for an agent wishing to forage to vote to move. Results, averaged (mean) over 1001 simulation runs using the adjusted range for $T_{F}$ and with occupancy penalty of $p=0.1$ are shown in Figure 2 .

As can be seen, the mean daily activity durations for the diminishing returns model are much closer to the observed values than those for the original simulation. In all habitats, utilisation time shows increased agreement with observed values, although as in [10] habitat time budgets remain characterised by an over-reliance on the vlei habitat.

We then analysed the data to determine the implications of the diminishing returns model for group decision making. In [10] a simulation was considered successful if all of the agents simultaneously achieved their targets for energy intake, water intake and time spent socialising over the entire simulated period and unsuccessful otherwise [10]. The targets used in [10] are shown in Table 2.

Table 2. Survival targets for agents

\begin{tabular}{ll}
\hline Attribute & Target \\
\hline Energy Intake & Approximately 3500kJ per day (dependant on activity pattern) \\
Water Intake & Agents must drink at least every two days \\
Social Activity & Agents must socialise for at least two hours per day \\
\hline
\end{tabular}




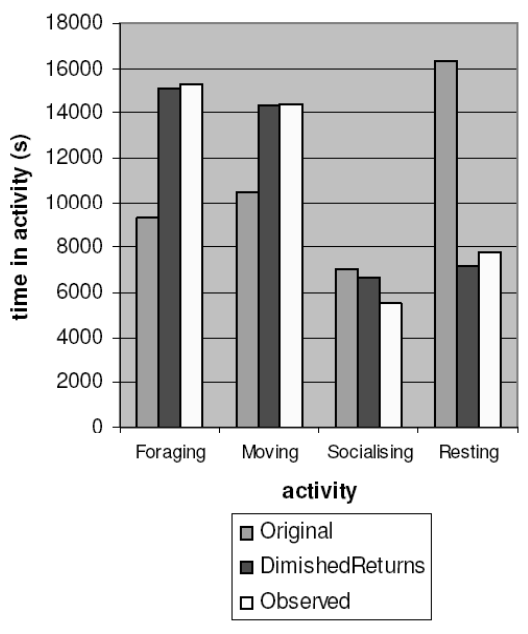

(a) Activities

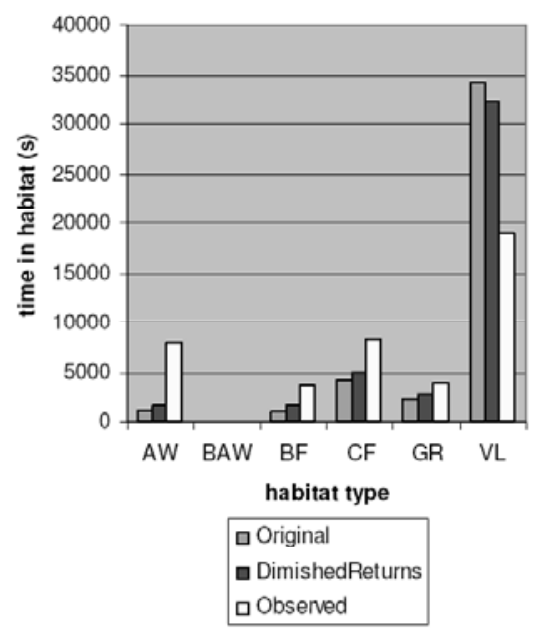

(b) Habitats

Fig. 2. Comparison of original, diminishing returns and observed mean daily activity and habitat utilisation times. Habitat types are given as: AW - Acacia Woodland, BAW - Burnt Acacia Woodland, BF - Burnt Fynbos, CF - Climax Fynbos, GR - Grassland, VL - Vlei.

A key finding from [10] was that the quorum threshold was the most important factor in determining if a run was successful, with values between 0.50 and $0.65(50 \%$ $65 \%$ ) yielding the greatest proportion of successful runs [10]. A comparison of the relationship between success and the quorum threshold for both simulations (see Figure 3) indicates that whilst this relationship remains, the introduction of diminishing returns has concentrated successful runs into the region where the quorum threshold lies between $55 \%$ and $65 \%$; with a noticeably pronounced peak in the region between $55 \%$ and $60 \%$.

\section{Discussion}

We have investigated the effects of resource availability on consensus decision making in primates groups. Through replication we have verified an existing agent-based model of primate group decision making [10] showing that, despite minor defects, the results of this model are not due to implementation artefacts. Further, we have extended the original work by providing a model of diminishing resource returns from foraging activity. We have shown that resource availability has a measurable effect on the quorum threshold necessary for successful group decision making. The introduction of diminishing returns also increased agreement between the predictions of the model and the observed behaviour of a naturally occurring primate group. 


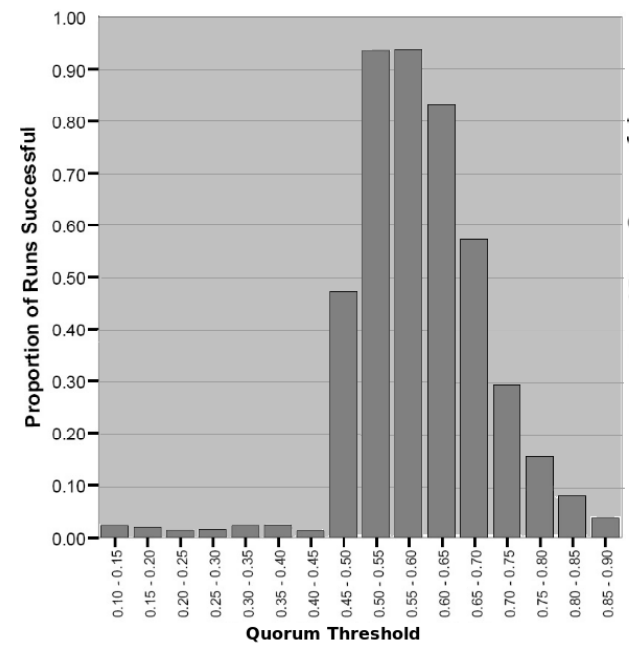

(a) Original

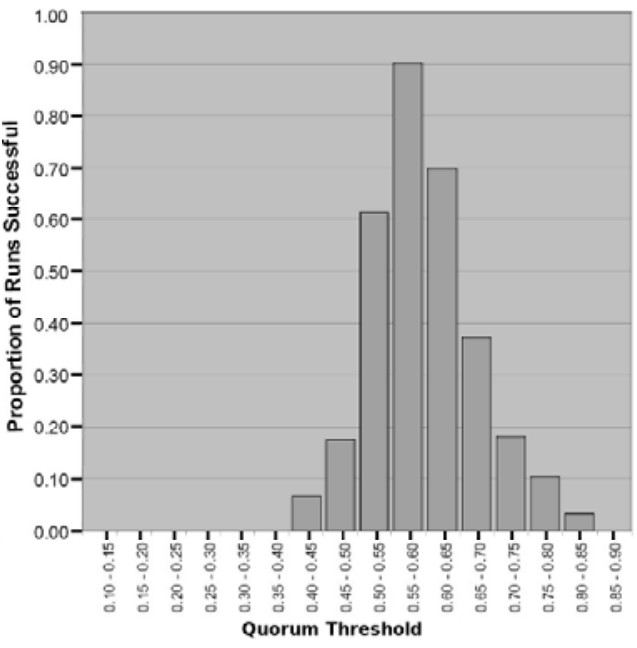

(b) New

Fig.3. Original \& new proportion of successful runs against the quorum threshold

It has been observed that "An unreplicated simulation is an untrustworthy simulation" [17]. The replication conducted during this work identified areas of the original model requiring improvement. Notably, deterministic group activity selection at submajority quorum thresholds may have been directly linked to the poor success of agents in simulations where this threshold is below 0.50 , a substantive finding of the original work. However, correction of this behaviour revealed that this is not the case; and although slight variation was introduced, the findings of Sellers et al. are supported by this work. The frame of reference provided by complete access to the original model was also instrumental in detecting, and correcting minor defects introduced whilst development of the new implementation was in progress; replication has undoubtedly contributed to the fidelity of both models.

The introduction of diminishing returns affects the model in a number of ways. The occupancy penalty decreases the rate at which agents can obtain food from a given location as a function of the time the spend at that location. This in turn requires that agents must increase the proportion of time spent foraging in order to achieve their energy intake target. Reducing the availability of food in cells previously occupied also discourages agents from revisiting foraging areas in the course of a simulated day, which, in turn, increases the proportion of time agents spend moving. Introduction of the occupancy penalty improves the fit between observed and simulated results, and tends to support the original hypothesis advanced in [10] that discrepancies between the Sellers et al. model and the field data were caused by the agents foraging too easily. 
Alteration to the environment model through introduction of the occupancy penalty also produced a noticeable effect on the proportion of successful simulation runs for a range of values of the quorum threshold. This suggests that environmental conditions, in this case resource availability, may influence the group decision making process, by playing a role in determining the value of the quorum threshold. The change observed in the relationship between the proportion of successful runs and the quorum threshold is attributed to changes in the environment introduced by the occupancy penalty. To the best of our knowledge, this relationship between resource (un)availability and 'successful' values of the quorum threshold has not been addressed in previous work. Although we have investigated only a small number of values for the occupancy penalty in the work presented here, our preliminary results suggest that as environmental conditions become less favourable, the range of quorum threshold values which result in the agents achieving their goals becomes smaller.

Our model of diminishing returns creates a less favourable environment for agents, consequently it is more challenging for them to achieve their survival goals. This change to the model of [10] is reflected in the differences found in the quorum response of the group (see Figure 3). We observe that sub-majority values for the quorum threshold produce noticeably fewer successful outcomes than found in the original model where diminishing returns are not present. In particular, the lower bound of the quorum threshold producing the maximum proportion of successful runs has increased from 0.50 (the majority threshold) to 0.55 (a super majority threshold) suggesting that greater commitment from the group is now required in order to prompt a change in activity. This behaviour is in agreement with the model of democratic decision making proposed by List [18], who shows that for the same group under differing circumstances, contrasting quorum thresholds may be required. Specifically, List suggests that, where food resource is scarce, a strategy of super majority decision making, as exhibited by our model, will reduce the probability of making the wrong decision.

The introduction of diminishing returns to the model of [10] also reduced the range of values for the quorum threshold producing successful outcomes for the agents, yet the process of democratic decision making employed in the model remains a stable strategy. Despite reduced resource availability agents were able to achieve their survival goals in a considerable proportion of the simulation runs (approximately 29\%). This result tends to confirm the view that democracy should be advantageous in a wide range of situations [3].

Recent empirical research supports the view that the group decision making process in primates can indeed be influenced by resource availability [4]. In this work, King et al. introduced artificial foraging patches to wild chacma baboon troops in the Tsaobis Leopard Park, Namibia. The distribution of food resource in these patches was designed to create a degree of resource contention amongst troop members. They found that, subject to social affiliation, resource contention can induce a group-fission event, where an otherwise stable naturally occurring group will divide into subgroups and follow independent foraging patterns. Such behaviour was not witnessed under normal foraging conditions.

In [19] Stueckle \& Zinner observed leadership and decision making in a troop of chacma baboons as they embarked on their morning departure in the De Hoop Nature 
Reserve, South Africa. Their findings indicate that a partially shared consensus decision was used to establish both the timing and direction of departure, and that leadership was distributed with most adult group members at some point initiating group movement. Stueckle \& Zinner note that this latter finding confirms behaviour predicted in [10] of similar baboons in a similar location suggesting that the decision making model proposed in [10] closely reflects the mechanism by which natural primate groups reach a consensus decision.

Field-based research conducted in $[4,19]$ both consider groups of chacma baboons, however these works reach different conclusions regarding the type of decision making utilised by their respective groups. Stueckle \& Zinner conclude that consensus is achieved through a partially shared process [19] whereas King et al. find that despotic (unshared) decision making is "the norm" [4]. These studies were conducted in different locations and presumably under diverse environmental conditions, the effects of which, as shown here, are pronounced and so may contribute towards this apparent disparity. We believe that agent-based modelling can be a useful approach to understanding such differences in group decision making in primates, as it permits fine-grained control of the entire system.

\section{Future Work}

Our current research qualitatively demonstrates some possible implications of resource availability on the group decision making process of primate groups. In future work we hope to extend this result through quantification of the relationship between resource availability and the quorum threshold. Results presented here lead us to speculate that introducing further granularity to resource availability within the environmental model will increase agreement between observed and simulated results. In particular, we expect that more accurate modelling of resource availability within the Vlei habitat type will redress the remaining discrepancies found in the agents' habitat utilisation.

In [10] group cohesion is enforced such that group fission events, as recorded in [4] are precluded. Our reimplementation of the original model was designed to permit migration towards a more individually oriented model. We anticipate that evolution of the model in this way will also provide opportunity to consider the effects of social and familial relationships upon the decision making process in primate groups.

\section{Acknowledgements}

We would like to thank Russell Hill for helpful comments on the diminishing returns model.

\section{References}

1. Hill, R., Lee, P.: Predation risk as an influence on group size in cercopithecoid primates: implications for social structure. Journal of Zoology 245 (1998) 447-456

2. Leca, J.B., Gunst, N., Thierry, B., Petit, O.: Distributed leadership in semifree-ranging whitefaced capuchin monkeys. Animal Behaviour 66 (2003) 1045-1052 
3. Conradt, L., Roper, T.: Group decision-making in animals. Nature 421 (2003) 155-158

4. King, A.J., Douglas, C.M., Huchard, E., Isaac, N.J., Cowlishaw, G.: Dominance and affiliation mediate despotism in a social primate. Current Biology 18 (2008) $1833-1838$

5. Conradt, L., Roper, T.: Democracy in animals: the evolution of shared group decisions. Proceedings of the Royal Society B: Biological Sciences 274 (2007) 2317-2326

6. Eberhardt, L., Thomas, J.: Designing environmental field studies. Ecological Monographs 61 (1991) 53-73

7. Milinski, M., Parker, G.A.: Competition for resources. In Krebs, J.R., Davies, N.B., eds.: Behavioural Ecology. Blackwell, Oxford (1991) 137-168

8. Pratt, S., Sumpter, D., Mallon, E., Franks, N.: An agent-based model of collective nest choice by the ant temnothorax albipennis. Animal Behaviour 70 (2005) 1023-1036

9. Garnier, S., Jost, C., Jeanson, R., Gautrais, J., Asadpour, M., Caprari, G., Theraulaz, G.: Aggregation behaviour as a source of collective decision in a group of cockroach-like-robots (2005)

10. Sellers, W., Hill, R., Logan, B.: An agent-based model of group decision making in baboons. Philosophical Transactions of the Royal Society B: Biological Sciences 362 (2007) 16991710

11. Conradt, L., Roper, T.J.: Consensus decision making in animals. Trends in Ecology \& Evolution 20 (2005) 449-456

12. Axelrod, R.: Advancing the art of simulation in the social sciences. Complexity 3 (1997) $16-22$

13. Luke, S., Cioffi-Revilla, C., Panait, L., Sullivan, K., Balan, G.: Mason: A multiagent simulation environment. Simulation 81 (2005) 517-527

14. Railsback, S., Lytinen, S., Jackson, S.: Agent-based simulation platforms: Review and development recommendations. Simulation 82 (2006) 609-623

15. Will, O., Hegselmann, R.: A replication that failed: On the computational model in'michael w. macy and yoshimichi sato: Trust, cooperation and market formation in the us and japan. proceedings of the national academy of sciences, may 2002'. Journal of Artificial Societies and Social Simulation (11) 3

16. Axtell, R., Axelrod, R., Epstein, J., Cohen, M.: Aligning simulation models: A case study and results. Computational \& Mathematical Organization Theory 1 (1996) 123-141

17. Edmonds, B., Hales, D.: Replication, replication and replication-some hard lessons from model alignment. Journal of Artificial Societies and Social Simulation (6) 11

18. List, C.: Democracy in animal groups: a political science perspective. Trends in Ecology \& Evolution 19 (2004) 168-169

19. Stueckle, S., Zinner, D.: To follow or not to follow: decision making and leadership during the morning departure in chacma baboons. Animal Behaviour 75 (2008) 1995-2004 